\title{
Auditory-visual coordination in infancy: Some limitations of the preference methodology
}

\author{
KEITH HUMPHREY \\ Queen's University, Kingston, Ontario K7L 3N6, Canada \\ and \\ RICHARD C. TEES \\ University of British Columbia, Vancouver, British Columbia V6T IW5, Canada
}

\begin{abstract}
Infants of 3,7 , and 10 months of age were placed midway between two visual displays that flashed on and off at two different temporal rates. Tones temporally synchronized to one of the visual displays emanated from concealed speakers placed midway between the visual displays directly in front of the infants. The visual displays and corresponding tones differed in temporal rate by a factor of four. No evidence was found for preferential looking to the sound-specified visual pattern in the 3-and 7-month-olds. Marginal evidence for such looking was found in the 10-month-olds. It is suggested that the results of the present study denote some of the limitations of the preference paradigm for the investigation of the development of auditory-visual coordination.
\end{abstract}

A number of recent studies have investigated sensitivity to acoustic-optical correspondences in infancy (see Mendelson, 1979, for a review). Much of the research has focused on the detection of common temporal or rhythmical relations between auditory and visual stimuli. For instance, Allen, Walker, Symonds, and Marcell (1977) habituated two groups of 7-month-old infants to a standard visual or auditory temporal sequence of light flashes or pure tones. Following habituation, infants showed greatest response recovery to a novel temporal sequence regardless of the sensory modality in which the temporal sequences were presented. Thus, by at least 7 months, infants are capable of perceiving equivalences and differences in temporal sequence information across modalities.

Another method for assessing infant sensitivity to temporal relations across modalities is a variation of the well-known preference paradigm devised by Spelke (1976). The modified procedure involves the simultaneous presentation of two motion picture films to infants. The films are separated, and the sound track emanates from a hidden speaker placed between the films. The infant is placed midway between the films in front of the concealed speaker. Measures of the infant's looking behavior are used to assess the sensitivity to the

This research was made possible by grants from the NSERC (PA-0179) and SSHRC (410-78-0222) of Canada to Richard C. Tees. A preliminary version of this report was presented at the 1980 meeting of the Canadian Psychological Association in Calgary, Canada. The authors thank A. J. Campbell and D. W. Muir for comments on an earlier draft of this manuscript. Requests for reprints should be sent to Richard C. Tees, Department of Psychology, University of British Columbia, Vancouver, British Columbia V6T 1W5, Canada. relationship between the sound track and the films. The rationale for the procedure and measurements is based on the assumption that information from one modality promotes exploration of information obtained from other modalities. For instance, an infant might seek visual information about auditory events to follow the events in two modalities. Research has shown that under such an experimental arrangement, 4-month-old infants will look at the film with an accompanying sound track more than at the simultaneously presented film without accompanying sound track (Spelke, 1976; Bahrick, Walker, \& Neisser, Note 1).

Spelke (1976) presented 4-month-old infants two motion picture films: One depicted a woman playing "peekaboo," and the other depicted a wooden baton striking a wood block or tambourine repeatedly and rhythmically. The results indicated that the infants looked primarily at the event specified by the relevant sound track. These results could have been obtained, however, if the infant associated the sound of the human voice with the motion picture of the woman-not an unlikely assumption, given the results of Spelke and Owsley (1979). Spelke and Owsley showed that, at least by 15 weeks of age, infants will search visually for a parent who is heard to speak in such a preference set-up. This occurs even when there is no spatial or temporal information uniting face and voice. In Spelke's (1976) study, it is conceivable that infants would look at the woman upon hearing the voice and at the "nonperson" otherwise, having learned that faces and voices go together. As such, it is unclear whether Spelke answered the question that prompted her research: "Will infants explore sights and sounds whose relationship is specified by their internal structure?" (Spelke, 1976, p. 554). 
Spelke's (1976) question has been answered more successfully by Bahrick et al. (Note 1). Bahrick et al. found consistent and strong preferences for soundrelated films by 4-month-old infants. The authors presented infants with every pairwise comparison of three films: a pattern of hand claps, the playing of a xylophone, and the movement of a slinky toy. Only the pairing of the xylophone and the slinky toy failed to reach statistical significance when the sound of the xylophone was presented. Although these results are not subject to the criticisms made of the Spelke (1976) report, the basis for detection of the temporal correspondences between the acoustic and optical events remains unspecified.

The present study was similar in design to those of Spelke (1976) and Bahrick et al. (Note 1), with an important exception: The number of stimulus dimensions was minimized by using simple light and sound stimuli. In the two displays, the pattern of visual elements and the sound that accompanied the patterns were the same in all respects, except for temporal rates. The stimuli were chosen for three reasons. First, the results of Allen et al. (1977) were obtained with relatively simple stimuli. Second, the physical characteristics of the stimuli could be fully specified. Third, there was little chance that infants would be familiar with the stimuli.

Three age levels were tested: 3,7 , and 10 months. These ages were chosen for three reasons. (1) Previous research has shown that infants as young as 2.5 months are sensitive to temporal sequence information within the auditory modality (Demany, McKenzie, \& Vurpillot, 1977). (2) In a recent review, McGurk and MacDonald (1978) suggested that systematic coordination between seeing and hearing begins to develop at about 3 months and develops rapidly thereafter. (3) These ages are representative of the three stages of intersensory differentiation outlined by Bower (1974). A sampling of these three ages could reveal developmental changes in intersensory coordination.

\section{METHOD}

\section{Subjects}

Forty-two infants participated in the experiment. Of the 42 , data for 6 were not fully collected due to equipment failure or excessive fussiness during testing. Three groups of 12 infants were tested, whose mean ages were 3 months 10 days (SD $=$ 12 days), 6 months 19 days ( $S D=19$ days), and 10 months 11 days ( $(S D=14$ days). Infants were recruited through advertisements in the local newspapers.

\section{Stimuli}

The visual stimuli were five light-emitting diodes (LEDs) embedded in clear plastic ( $5 \mathrm{~cm}$ apart) and arranged in a cross pattern. Whenever the visual pattern received an electronic pulse, the five diodes were illuminated simultaneously. The visual angle subtended by the pattern was approximately $7.2 \mathrm{deg}$. A red translucent plastic sheet was placed over the diodes to enhance their brightness. Two such visual stimulus patterns, separated by $70 \mathrm{~cm}$, were used; one was placed to the right and the other was placed to the left of the infant. A green fixation light was placed midway between the visual patterns. The visual angle between a visual pattern and the fixation light was approximately $26 \mathrm{deg}$. Auditory signals were produced by commercially available sonalerts (Model SC628), manufactured by P. R. Mallory Company, Inc., with a nominal frequency of 2,900 \pm $500 \mathrm{~Hz}$. Two sonalerts were concealed and placed midway between the visual panels. Ambient noise level, recorded at the infant's head and measured by a General Radio Company sound level meter (Model 1551C), was 52dB. The sound level was $62 \mathrm{~dB}$ with the tone present.

The pulse rates were generated by a specially constructed two-channel pulse generator. Two temporal rates were controlled by separate channels of the generator. The cycle time for the slow-tempo pattern was $1.4 \mathrm{sec}$, with the stimuli on for $1.0 \mathrm{sec}$ and off for $.4 \mathrm{sec}$. That is, during a trial, the visual pattern continually cycled, so that it was on for $1.0 \mathrm{sec}$ and off for $.4 \mathrm{sec}$ repeatedly. For the fast-tempo pattern the cycle time was $.35 \mathrm{sec}$, with a $.25-\mathrm{sec}$ on period and $10-\mathrm{sec}$ off period. Thus, the fast-tempo pattern cycled at a rate four times faster than the slow temporal pattern. During any one trial, one visual stimulus had a fast tempo and the other had a slow tempo; the simultaneously presented auditory stimulus was temporally synchronous with one of the visual patterns.

\section{Procedure and Design}

Testing took place in a specially constructed darkened enclosure $(1.2 \times 1.3 \times 1.9 \mathrm{~m})$ in a dimly lit, quiet room. The infants were held by the mother at a distance of approximately $80 \mathrm{~cm}$ from a point midway between the visual panels. The infants were viewed by an observer through a peephole midway between the visual panels. Both the mother and the observer heard music through headphones that masked the auditory stimulus throughout the testing session.

The observer began a trial when the infant oriented to the green fixation light. A light was used to inform the observer of a trial's end. Trial duration was controlled by a Lafayette VIII bank timer (Model 5431A) and was $60 \mathrm{sec}$. Whenever the infant was judged to be looking at a visual panel during a trial, the observer depressed one of two switches, one switch for looking at the right panel and the other for looking at the left. The switches activated separate pens on a Rustrak event recorder (Model 92).

Each infant received two trials with the fast-tempo auditory pattern and two trials with the slow-tempo auditory pattern. The order of fast and slow patterns was randomized across infants. Half of the infants in each group had the fast-tempo visual pattern on their right and the slow-tempo visual pattern on their left; the other half were presented the opposite arrangement.

\section{RESULTS AND DISCUSSION}

Two response biases required treatment prior to the general analysis. First, a significant proportion of looking toward the right in the 3 -month-old group was different from chance [i.e., .50; $\mathrm{t}(11)=2.30, \mathrm{p}<.05$, two-tailed test]. Position bias has been reported in previous studies that have used measures of visual preference (Caron, Caron, Minichiello, Weiss, \& Friedman, 1977; Cohen, 1976; Ruff \& Birch, 1974). Second, the 10-month-ald group looked at the visual pattern with the faster temporal rate more often regardless of position or tempo of the accompanying sound $[t(11)=3.06$, $\mathrm{p}<.02$, two-tailed test] .

To illustrate the correction for bias, consider, for 
example, the visual tempo bias exhibited by the 10 month-olds. The correction simply involved calculating the proportion of looking toward the fast-tempo visual pattern during trials in which the fast-tempo sound was present and, similarly, for the slow-tempo sound conditions. If the infant's looking was totally uninfluenced by the tempo of the sound, then looking should have been approximately evenly distributed (i.e., .50) during the two sound conditions. However, if the sound did influence looking, then one could expect a departure from evenly distributed looking.

The use of such a correction procedure made it possible to detect an increased proportion of looking toward a sound-related visual pattern, even if there was a strong bias to look in just one direction or at one visual pattern. The procedure was used for both position and visual tempo biases. (Although the 7-month-olds showed no systematic bias, data from all three groups were corrected in the same manner for purposes of comparison.) Two $t$ tests were performed on the data for each age group, one for the slow-tempo condition and one for the fast. In each case, the corrected proportion of looking time to the sound-related visual stimulus was compared with the proportion expected by chance (i.e., .50).

The results are presented in Table 1 . As can be seen from the table, the results for the 3-and 7-month-olds clearly did not reach statistical significance. The results for the 10-month-olds were marginally significant.

These results suggest that preferential looking toward sound-related visual events is absent, at least at 3 and 7 months of age, when stimuli differ in tempo only. Spelke (1979) has also found that preferential looking is reduced relative to the results obtained in other research (Spelke, 1976; Bahrick et al., Note 1) when the visual events are fairly similar to each other but differ in tempo. Spelke (1979) did obtain evidence for sensitivity to the auditory-visual correspondence with alternate measures of "visual search." The measures included, over a number of trials, direction of first look, latency of first look, duration of looks to both the synchronized and the nonsynchronized film (within $5 \mathrm{sec}$ after stimulus onset), and the number of trials on which the infant looked at all to the synchronized or nonsynchronized films.

Table 1

Corrected Proportion of Looking Time (P) to the Sound-Appropriate Visual Pattern

\begin{tabular}{cccccc}
\hline \multirow{2}{*}{$\begin{array}{c}\text { Age (in } \\
\text { Months) }\end{array}$} & \multicolumn{2}{c}{ Fast Tempo } & & \multicolumn{2}{c}{ Slow Tempo } \\
\cline { 2 - 3 } \cline { 5 - 6 } & $\mathrm{P}$ & $\mathrm{t}(11)$ & & $\mathrm{P}$ & $\mathrm{t}(11)$ \\
\hline 3 & .54 & .72 & & .46 & .46 \\
7 & .57 & 1.04 & & .50 & .05 \\
10 & .58 & $2.69^{*}$ & & .57 & $1.64 \dagger$ \\
\hline
\end{tabular}

${ }^{*} p<.025$, one-tailed test. $\quad \dagger_{p}<.07$, one-tailed test.
Bahrick et al. (Note 1) have argued that the attenuated preference effect reported by Spelke (1979) may have resulted from the simplicity of the stimuli. They argue that either the infants were able to follow both events or the infants found such simple events to be uninteresting. By implication, then, studies demonstrating strong preferential looking to sound-related visual events result from the complexity and/or higher interest generated by the stimuli employed. An alternate explanation for the preferential looking demonstrated by Spelke (1976) and Bahrick et al. (Note 1) is that the stimuli were not as difficult to discriminate. That is, the stimuli used by Spelke (1976) and Bahrick et al. (Note 1) were not necessarily more interesting, but they were easier to discriminate.

The stimuli in the studies of Spelke (1976) and Bahrick et al. (Note 1) were more complex than those used in the present study and in the Spelke (1979) report. For instance, the visual events differed in color, form, and type of movement, whereas the accompanying sounds differed in spectral frequency composition. Furthermore, the rhythmic relationship between the auditory and visual stimuli was complex and much less repetitive than in either the present study or the report of Spelke (1979).

Interpreting Spelke's (1979) results in terms of the difficulty of the discrimination rather than in terms of the inherent interest of the items is supported by the behavior of the 10-month-olds in the present study. Recall that the 10-month-olds (presumably, they are better "perceivers" than both the 3-and 7-month-olds) were the only age group to show a marginal preference. Thus, decreasing the number of differences between the stimuli made the task more difficult rather than uninteresting.

Regardless of the reasons for lack of preferential responding in the present experiment, it is clear that the preference technique becomes less sensitive under conditions that minimize the differences between visual and auditory stimuli. Thus, the present experiment provides limits for the preference technique as a method assessing auditory-visual coordination in infancy. Perhaps measures of visual search would have been more revealing. Nevertheless, we attribute the lack of success in demonstrating sensitivity to simple auditory-visual events to the technique.

Humphrey, Tees, and Werker (1979) have shown, using a habituation methodology, that 4-month-olds are very sensitive to the phase relationship between light and sound patterns similar to those employed in the present study. Hence, lack of differential looking cannot be attributed to a lack of sensitivity to such auditoryvisual relationships, even at 4-months-of age.

As Caron et al. (1977) have pointed out, our assessment of the young infants' capacities has largely been the result of methodological advances. A continuous 
evaluation of these procedures is necessary, however, to maintain a reasonable estimate of the young infants' competence. Such continuous evaluation requires openness to new techniques, such as that devised by Spelke (1976), as well as a willingness on the part of experimenters to explore the limits of such techniques.

\section{REFERENCE NOTE}

1. Bahrick, L. E., Walker, A. S., \& Neisser, U. Infants' perception of intermodal relationships in natural events. Paper presented at the meeting of the Eastern Psychological Association, Washington, D.C., 1978.

\section{REFERENCES}

Allen, T. W., Walker, K., Symonds, L., \& Marcell, M. Intrasensory and intersensory perception of temporal sequences during infancy. Developmental Psychology, 1977, 13, 225-229.

Bower, T. G. R. Development in infancy. San Francisco: Freeman, 1974.

Caron, A. J., Caron, R. F., Minichiello, M. D., Weiss, S. J., \& FrIEDMAN, S. L. Constraints on the use of the familiarizationnovelty method in the assessment of infant discrimination. Child Development, 1977, 48, 747-762.
Conen, L. B. Habituation of infant visual attention. In T. J. Tighe \& R. N. Leaton (Eds.), Habituation: Perspectives from child development, animal behavior and Neurophysiology. Hillsdale, N.J: Erlbaum, 1976.

Demany, L., McKenzie, R., \& Vurpillot, E. Rhythm perception in early infancy. Nature, 1977, 266, 718-719.

Humphrey, K., Tees, R. C., \& Werker, J. Auditory-visual integration of temporal relations in infants. Canadian Journal of Psychology, 1979, 33, 347-352.

McGurk, H., \& MacDonald, J. Auditory-visual coordination in the first year of life. International Journal of Behavioural Development, 1978, 1, 229-240.

Mendelson, M. J. Acoustic-optical correspondence and auditoryvisual coordination in infancy. Canadian Journal of Psychology, 1979, 33, 334-346.

Ruff, H. A., \& Birch. H. G. Infant visual fixation: The effect of concentricity, curvilinearity, and number of directions. Journal of Experimental Child Psychology, 1974, 17, 460-473.

SPElKe, E. S. Infant's intermodal perception of events. Cognitive Psychology, 1976, 8, 553-560.

Spelke, E. S. Perceiving bimodally specified events in infants. Developmental Psychology, 1979, 15, 626-636.

SPelKe, E. S., \& OWsley, C. J. Intermodal exploration and knowledge in infancy. Infant Behavior and Development, 1979, 2, 13-27.

(Received for publication July 17, 1980.) 